\title{
REPUTACIÓN ONLINE Y USO DE LA WEB 2.0 EN LOS HOTELES DE LA CIUDAD DE CÓRDOBA (ESPAÑA)
}

\section{ONLINE REPUTATION AND WEB 2.0 IN THE HOTELS OF CORDOBA (SPAIN)}

Pablo M. Cañero Morales ${ }^{1}$ Universidad ULH - Haití pablo canero@ulh.edu.ht

Salvador Moral Cuadra ${ }^{2}$ Universidad ULH - Haití smoralcuadra@ulh.edu.ht

\author{
Francisco Orgaz Agüera ${ }^{3}$ \\ Universidad de UTESA - República Dominicana
} franorgaz@utesa.edu

(Fecha envío: 14/03/15 - Fecha aceptación: 21/05/15)

\begin{abstract}
Resumen
Internet se ha convertido en un elemento fundamental para cualquier actividad económica, aunque requiere para ello una actualización constante debido a sus diversos cambios. Así, dentro del turismo, ha aparecido lo que se conoce como web 2.0, permitiendo la interacción directa entre diferentes empresas, organizaciones y/o usuarios, sobre todo, a partir de las redes sociales. En este sentido, y dentro del sector turístico, los hoteles ocupan una posición destacada, debido a su alta interrelación con Internet. Este artículo tiene como objetivo analizar una serie de factores relacionados con la presencia de los hoteles de la ciudad de Córdoba (España) en lo que se conoce como web 2.0 (redes sociales, opiniones de la demanda turística, etc.) y la medida de su reputación online. La metodología utilizada para el desarrollo de este estudio ha consistido en una revisión de la literatura científica y una recolección de datos de redes sociales y de la página de viajes TripAdvisor.
\end{abstract}

\section{Palabras clave}

hoteles, reputación online, redes sociales, TripAdvisor, Córdoba

\begin{abstract}
Internet has become a key element in any economic activity, but to do so requires constant updating due to its various changes. Thus, within tourism, has appeared what is known as Web 2.0, allowing direct interaction between different companies, organizations and/or users, especially from social networks. In this sense, and in the tourism sector, hotels are the ones with the strongest position, because of their high interaction with internet. The purpose of this study is to analyze a series of factors related to the presence of the hotels in the city of Cordoba (Spain) in what is known as Web 2.0 (social networks, online reputation, reviews of tourist demand, etc.) and the extent of their online reputation. The methodology used for this research consisted in a review of similar written studies in other tourist destinations, and data collection in social media and website of TripAdvisor.
\end{abstract}

\section{Keywords}

hotels, online reputation, social media, Trip Advisor, Cordoba

\footnotetext{
1 Profesor en Turismo de la Universidad Libre de Haití. Doctorando en Ciencias Sociales y Jurídicas en la Universidad de Córdoba (España). Máster en Dirección y Planificación del Turismo por la Universidad de Sevilla (España). Su principal línea de investigación es el turismo.

2 Profesor en Turismo de la Universidad Libre de Haití. Máster en Dirección y Planificación del Turismo por la Universidad de Sevilla (España). Su principal línea de investigación es el turismo.

3 Profesor en Turismo y Administración de Empresas en la Universidad Tecnológica de Santiago (UTESA) de República Dominicana. Doctor en Turismo por la Universidad de Sevilla (España). Máster en Dirección y Planificación del Turismo por la Universidad de Sevilla (España). Su principal línea de investigación es el turismo.
} 


\section{INTRODUCCIÓN}

En la globalización actual, Internet está tomando un papel muy importante, no solo a la hora del intercambio de información entre las diferentes partes del mundo, sino a la hora de comercializar productos y servicios de cualquier índole, entre ellos los turísticos (Conde et al., 2011; Domínguez y Araujo, 2012). Así, el turismo es un sector económico que debe ser abordado de una manera especial debido a la interconexión de todos sus agentes locales involucrados en las actividades turísticas, y en este caso, se debe tener presente que muchos de esos stakeholders se manifiestan en Internet.

Desde que apareció Internet, las empresas del sector turístico han estado muy ligados a él (Martínez, 2011) por las numerosas ventajas que esta herramienta tenía para la comunicación (Luque y Castañeda, 2007). Según Devis-Botella (2010), la importancia de Internet en el sector turístico se debe a que a través de esta nueva herramienta, cada usuario puede decidir el destino final, en qué hoteles desea alojarse o que actividades desea llevar a cabo en el área geográfica, todo ello a través de una pantalla conectada a Internet (Martínez et al., 2013). Es decir, lo que antes hacía solo una minoría, ahora lo realiza la gran mayoría, por lo que, gracias al fenómeno Web 2.0 esta tendencia ha cambiado y toma cada vez más importancia los recursos electrónicos para conformar el viaje (Lincoln, 2009). Por tanto, la Web 2.0 se conforma como una herramienta básica y muy importante a la hora de dar a conocer y ofrecer los servicios y productos por parte de las empresas en los destinos geográficos, adoptando una postura de diálogo y transparencia con el cliente, un enfoque proactivo con éste, conociéndose esto como reputación online (Del Fresno, 2011). Centrándonos en el caso de los hoteles, además de tener una página web para comercializar su servicio, deben decidir en qué red social tienen más presencia para interactuar con el cliente y en qué red social suben videos y fotos para promocionar sus servicios (Martínez et al., 2013). También cabe resaltar, que Internet ha contribuido a mejorar el aprendizaje organizacional en las grandes cadenas hoteleras (Rodríguez et al., 2008).

El objetivo de esta investigación consiste en analizar una serie de factores relacionados con la presencia de los hoteles de la ciudad de Córdoba (España) en lo que se conoce como Web 2.0 (redes sociales, opiniones de la demanda turística, etc.) y la medida de su reputación online. Se pretende observar si las redes sociales y las categorías de estrellas influyen en la reputación online de los hoteles. En este caso se analizan las redes sociales de los hoteles y los datos obtenidos sobre los hoteles en el portal web TripAdvisor. La metodología utilizada para realizar esta investigación ha consistido en una revisión de la literatura de estudios similares en otros destinos turísticos, y una recolección de datos de redes sociales (Facebook y Twitter) y página de TripAdvisor.

Para cumplir con estos objetivos, este artículo se estructurará tras esta introducción, en un segundo apartado que tratará sobre el marco teórico del tema estudiado. Posteriormente, en el tercer apartado se realizará una descripción del destino geográfico, para posteriormente, en un cuarto apartado, tratar la metodología de esta investigación. En un quinto apartado se mostrarán los resultados de este estudio, para a posteriori, en un sexto apartado realizar las principales conclusiones de este artículo. Finalmente, se muestran las referencias bibliográficas utilizadas por los autores.

\section{REVISIÓN DE LA LITERATURA}

El marco teórico de esta investigación lo hemos dividido en dos partes. La primera versa sobre la Web 2.0 y su relación con el turismo, y la segunda, sobre la reputación online y TripAdvisor.

\subsection{Las Web 2.0 y el turismo}

Según O'Reilly (2005), se puede entender por Web 2.0 el conjunto de aplicaciones que tratan de abarcar la red entendiendo cómo funcionan los efectos de la red y aprovechándolos en todo lo que se hace. Así, aunque actualmente no existe una definición consensuada acerca de este término, este autor fue pionero en definir el concepto (O'Connor, 2008). En este sentido, según Lincoln (2009), con la aparición de la Web 2.0 nos encontramos de nuevo ante un período en el que las organizaciones deben decidir si apostar por la innovación en Internet o, por otro lado, esperar. Según Martínez et al. (2012) anteriormente en el mundo hotelero la pregunta era si creaban o no una web, y ahora, la pregunta es si comentan en Facebook o en Twitter, si suben videos a Youtube o a Vimeo, o si suben fotos a Flickr o Instagram. En este contexto nace el concepto conocido como Turismo 2.0, que consiste básicamente en el uso de las herramientas de la Web 2.0 por parte de los usuarios de productos y servicios turísticos, teniendo una especial importancia los comentarios de los turistas sobre sus experiencias de viaje compartiendo fotos o vídeos (Martínez et al., 2013). Por esta razón, la demanda turística se ve influenciada por las opiniones de otros viajeros que se vierten en las numerosas redes sociales (Gretzel y Yoo, 2008; Vermeulen y Seegers, 2009; Martínez, 2011) a la hora de elegir un hotel para sus estancias en los destinos. Según Dellarocas (2003), esto también se conoce como "boca a boca" adaptado a Internet. De esta forma, cabe destacar que la presencia en redes sociales de los hoteles ha sido objeto de investigación en los últimos años (Callarisa et al., 2012; Hsu, 2012; Bulchand-Gidumal et al., 2013; Escobar-Rodríguez y CarvajalTrujillo, 2013). 
Siguiendo a Domínguez y Araujo (2012), el fenómeno 2.0 engloba herramientas (blogs, redes sociales, galerías de imágenes, etc.) necesarias para que la empresa sobreviva y llegue a sus clientes potenciales. Así, resulta vital el "estar conectado", siendo un proceso que se ha interiorizado y del cual dependen multitud de personas, organismos o empresas, dejando de ser, por tanto, una mera tendencia pasajera (Kahn et al., 1997). En este sentido, las áreas de marketing de las empresas turísticas han sido las que más se han beneficiado de la Web 2.0 (López et al., 2009). Según Rivero (2006), entre las principales ventajas o beneficios de las Web 2.0 como elemento para el marketing caben destacar las siguientes: accesibilidad, comodidad, rapidez, novedad, cobertura global, gran riqueza, flexibilidad y reducido coste (Hijazi, 2002; Martínez, 2011). Para Murolo (2009), todo esto se debe a que hoy día se puede consumir Internet en cualquier parte del mundo y con diversos dispositivos, que van más allá del ordenador, como son los teléfonos móviles, las PDAs o tablets. Fumero y Roca (2007) destacan la importancia de las Web 2.0, y en especial al uso de redes sociales, que ponen de manifiesto que existe un gran número de usuarios que actualmente utilizan estos medios de comunicación. Así, las redes sociales son tan importantes que algunas de ellas han sido analizadas para conocer la imagen turística de un destino específico (Donaire y Galí, 2011). Aunque para Gutiérrez (2005), también existen inconvenientes por la utilización de Internet y las redes sociales en el turismo.

Para Fumero y Roca (2007), se puede diferenciar 21 agrupaciones diferentes dentro de este "Universo 2.0": redes sociales personales, compartir fotos, microblogging, podcasting, compartir vídeos, aplicaciones sobre mapas, redes sociales temáticas, agregadores, ranking de weblog y herramientas, buscadores 2.0, páginas de inicio personalizadas, redes de blog, redes sociales profesionales, comunidades de blogs y directorios, redes sociales de movilidad y mapas, video-blogs, mercados sociales, noticias y contenidos votados por usuarios, sistemas de creación y alojamiento, wikis, y por último, lectores RSS y servicios relacionados. En el caso de los hoteles, estos necesitan cada vez más información para su correcta gestión y para maximizar su desempeño o éxito empresarial (Claver et al., 2007). Por tal razón, estos alojamientos han empezado a incorporar nuevas herramientas en línea tales como los medios de comunicación social con la misión de mantener relaciones más estrechas con los clientes y los inversores, aunque en España generalmente se dirigen a los clientes (EscobarRodríguez y Carvajal-Trujillo, 2013). Cabe resaltar que cada día aumenta más el volumen de contenido generado por el cliente en las plataformas webs de medio sociales (Callarisa et al., 2012). En este sentido, los hoteles que no tienen redes sociales como Facebook son peor valorados que los que sí tienen en los portales webs, al igual que aquellos tienen redes sociales independientes a la cadena hotelera tienen valoraciones más altas en los portales webs (Hernández et al., 2012; Martínez et al., 2012). En este aspecto, Facebook se está consolidando como una de las redes sociales que ofrece más oportunidades para desarrollar acciones marketing online para los hoteles (Hsu, 2012), consolidándose como la principal red social para los hoteles (Martínez et al., 2013).

\section{H1: Facebook se consolida como la principal red social empresarial.}

H2: Los hoteles que no tienen Facebook son peor valorados que los que sí tienen.

H3: Los hoteles independientes de cadenas hoteleras tienes valoraciones más altas que los hoteles pertenecientes a una cadena hotelera.

\subsection{La reputación online}

La reputación es un concepto que ha sido objeto de numerosos estudios desde finales del pasado siglo XX, si bien se trataba de un concepto estudiado desde un punto de vista tradicional. Así, para Waddock (2000), la reputación corporativa es la capacidad percibida de la organización para satisfacer las expectativas de sus stakeholders. Según Gray y Balmer (1998), se define como la valoración de los atributos de la empresa según sus grupos de interés. En España, para el Foro de Reputación Corporativa (2005), la reputación hace referencia al conjunto de percepciones que tienen sobre la empresa los diversos stakeholders con los que se relaciona, tanto internos como externos.

Sin embargo, la forma en que se construye y gestiona la reputación ha cambiado con la generalización de Internet y las redes sociales, tanto a nivel general como específicamente en el sector turístico y hotelero, apareciendo un concepto de reputación mucho más moderno y actualizado a los nuevos tiempos. Hablamos de la reputación online, que viene siendo objeto de estudio en los últimos años para algunos sectores (Park y Lee, 2007; Caruana y Ewing, 2010; Aula, 2011; Liu y Munro, 2012), incluido en el turismo (Cao y Schniederjans, 2006; Kim et al., 2011; Chen et al., 2013; Zeng y Gerritsen, 2014). Así, para Antón (2008), es difícil adaptar correctamente el concepto de reputación corporativa a su versión online porque la reputación de una empresa en Internet no es equivalente a la reputación total de la empresa. Siguiendo a Vaquero (2012), el concepto de reputación online nace como resultado de las buenas prácticas empresariales en Internet y el buen comportamiento corporativo en la doctrina del management. Del Fresno (2012) la define como el resultado de lo que clientes, ex-clientes, futuros clientes, empleados, etc. dicen, escriben y transmiten a otros en cualquier parte de los medios sociales de Internet o redes sociales a partir de sus percepciones y experiencia en cualquier momento de su relación, directa o indirecta con esa marca. Antón (2008) afirma que la reputación online se puede definir como la valoración alcanzada por una empresa a través del uso o mal uso de las posibilidades que ofrece Internet. Por tanto, los conceptos anteriores giran en torno a la importancia de la gestión de la reputación online como una herramienta de marketing en Internet (Hung et al., 2012). 
Por otro lado, la comunicación corporativa a través de las webs de las empresas es un factor clave en la construcción de esta reputación online. A partir de la orientación y características de los contenidos en estas webs se facilitan una relación posterior con la empresa según el consejo, búsqueda o navegación que recibe un internauta ocasional (Álvarez et al., 2010). Las conversaciones realizadas a través de blogs, de las wikis y en las redes sociales aportan dos tercios del renombre corporativo, además de no afectar de la misma manera a todas las empresas (Celaya, 2008).

Por su parte existen diversos portales webs para buscar hoteles, y poder realizar y observar las críticas y valoraciones otorgadas a un hotel en concreto por parte de los turistas que se han alojado en él. Algunos de los más importantes son TripAdvisor y Booking, aunque también existen otros como Atrápalo, Rumbo, Edreams o Trivago. En este sentido, TripAdvisor ha sido uno de los portales más estudiados por la comunidad científica (Law, 2006; O'Connor, 2008; Miguéis et al., 2008; Jeacle y Carter, 2011; Vásquez, 2011; Ayeh et al., 2013; Bulchand-Gidumal et al., 2013; Lee et al., 2013; Levy et al., 2013). Así, a través de esta web, el turista accede para planificar su viaje, debido a que ahí puede encontrar valoraciones de otros turistas respecto a los hoteles del destino y conocer también que otras atracciones turísticas existen cerca de los hoteles (Miguéis et al., 2008). Actualmente, TripAdvisor cuenta con más de 50 millones de visitas al mes y aproximadamente 60 millones opiniones (Hernández et al., 2012). TripAdvisor funciona como foros de discusión donde se muestran valoraciones y opiniones de otros turistas. Para Levy et al. (2013) las valoraciones de los turistas no influyen en la decisión de otros viajeros a la hora de elegir un destino, poniendo el ejemplo de un estudio comparado a través de TripAdvisor entre los hoteles de un destino consolidado (Las Vegas) y otro no consolidado (Irlanda). Según Callarosa et al. (2012), mediante estas plataformas webs los turistas escriben la opinión y satisfacción sobre su estancia en el hotel, lo cual hace que la empresa pueda conocer que piensan los usuarios sobre los servicios recibidos y como pueden mejorar, eliminando la realización de otras técnicas que tiene como objetivo conocer la percepción del turista sobre el servicio, como es el caso de las encuestas rápidas. Cabe destacar que TripAdvisor, además de tener opiniones y valoraciones de los viajeros, también ofrece la posibilidad de reservar un hotel a través de links de algunos proveedores (Hotels.com, Booking.com, Kayak.com, etc.). En este sentido, y según Hernández et al. (2012), las categorías de estrellas de los hoteles influyen en la puntuación media otorgada por los clientes.

H4: La categoría de estrellas de los hoteles influye en la puntuación media otorgada por los clientes en los portales webs.

\section{DESCRIPCIÓN DEL ÁREA GEOGRÁFICA}

El área geográfica analizada para este estudio ha sido la ciudad de Córdoba (mapa 1), localizada al sur de España, en la región de Andalucía. Esta ciudad, debido a su importancia patrimonial y cultural fruto de las civilizaciones que han ido dejando huella a lo largo de los siglos, se ha convertido en una de los destinos geográficos más importantes en lo que a patrimonio cultural se refiere, recibiendo al año un gran número de turistas.

Figura 1. Localización de la ciudad de la ciudad de Córdoba en la región de Andalucía.

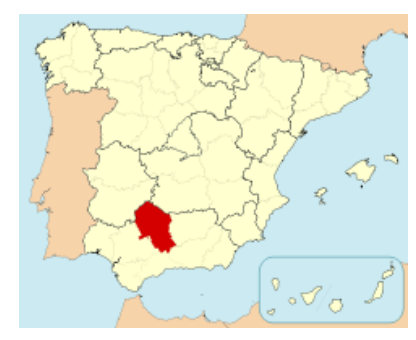

Fuente: Google imágenes

Teniendo en cuenta lo anterior, han sido muchas las tipologías turísticas que se han investigado en este destino geográfico (tabla 1).

Tabla 1. Tipologías turísticas investigadas en Córdoba

\begin{tabular}{|r|l|}
\hline \multicolumn{1}{|c|}{ Tipología Turística } & \multicolumn{1}{c|}{ Algunos Autores } \\
\hline Turismo gastronómico & López-Guzmán y Sánchez (2012); Millán et al. (2012) \\
\hline Turismo idiomático & Piédrola y Artacho (2011) \\
\hline Turismo rural & Millán y Agudo (2013) \\
\hline Turismo cultural & Ortiz (2013) \\
\hline Turismo arqueológico & Morena (2011) \\
\hline Oleoturismo & Millán et al. (2014); Moral et al. (2014) \\
\hline
\end{tabular}




\begin{tabular}{|r|l|}
\hline Enoturismo & López-Guzmán et al. (2009) \\
\hline Ecoturismo & López-Guzmán et al. (2007) \\
\hline Turismo Industrial & Castillo et al. (2010) \\
\hline
\end{tabular}

Fuente: Elaboración propia.

En todo caso, esta literatura académica deja clara la importancia del turismo en este destino andaluz y, por tanto, han dado lugar a la aparición de otras investigaciones en el destino centradas en el análisis de las tipologías turísticas más importantes para Córdoba (Moral y Orgaz, 2014) y en el sector hotelero (Sánchez et al., 2007; González et al., 2011). Según la web de turismo de Córdoba (http://www.turismodecordoba.org) existe un total de 53 hoteles en la ciudad, siendo uno de cinco estrellas, 16 de cuatro estrellas, nueve de tres estrellas, 19 de dos estrellas y ocho de una estrella. Además, existen un gran número de hostales y pensiones, campings, alojamientos rurales, apartamentos turísticos y albergues.

Por tanto, aunque se han estudiado una gran parte de las tipologías turísticas de Córdoba y sus potenciales para otras, y a su vez, también se han analizado algunos elementos referentes a los hoteles, con este artículo se contribuye al análisis del turismo en Córdoba desde la óptica de la reputación online de los alojamientos hoteleros.

\section{METODOLOGÍA DE LA INVESTIGACIÓN}

En esta investigación se ha planteado la hipótesis H1 (Facebook se consolida como la principal red social empresarial), H2 (los hoteles que no tienen Facebook son peor valorados que los que sí tienen), H3 (los hoteles independientes de cadenas hoteleras tienes valoraciones más altas que los hoteles pertenecientes a una cadena hotelera) y H4 (la categoría de estrellas de los hoteles influye en la puntuación media otorgada por los clientes en los portales webs). Para observar si estas hipótesis son soportadas, la investigación que aquí se presenta ha utilizado una metodología basada en fuentes secundarias, a través de la recogida de datos del portal web de TripAdvisor y de las redes sociales Facebook y Twitter. Además, se ha realizado una revisión de la literatura de estudios relacionados en otros destinos turísticos y sobre el tema analizado.

De esta forma, se han seleccionado los hoteles de tres, cuatro y cinco estrellas de la ciudad de Córdoba, siguiendo la página web de turismo y teniendo en cuenta otros estudios (Martínez et al., 2012) que también han escogido los hoteles de estas categorías de estrellas porque representan las categorías medias-altas de hoteles en un destino. En total, hay una población de 26 hoteles, repartidos en un (1) hotel de cinco estrellas, dieciséis (16) hoteles de cuatro estrellas y nueve (9) hoteles de tres estrellas. Así, la muestra selecciona para realizar el análisis ha sido el total de la población de hoteles de tres, cuatro y cinco estrellas de la ciudad de Córdoba (tabla 3).

Para cada uno de los hoteles estudiados se han analizado 11 parámetros diferentes, adaptándolos siguiendo la literatura científica (Martínez et al., 2012; Hernández et al., 2012; Martínez et al., 2013). Estos parámetros versan sobre las características básicas de cada hotel, la intensidad de su presencia en Internet, el aprovechamiento de las herramientas que proporciona la web, su integración en la Web 2.0, su reputación online y la valoración de los usuarios sobre estos hoteles en la red. La búsqueda de estos datos se ha desarrollado durante el mes de mayo de 2014.

A continuación, en tabla 2 se muestran las variables concretas analizadas para la investigación presentada en este artículo, mostrándose el número de variable, las propias variables analizadas y la subclasificación de las mismas.

Tabla 2. Variables analizadas

\begin{tabular}{|c|c|c|}
\hline № Variable & Variable Analizadas & Subclasificación \\
\hline 1 & Número de estrellas & \\
\hline 2 & Número de fotos en la web & \\
\hline 3 & Número de vídeos en la web & \\
\hline 4 & Tipo de web & $\begin{array}{c}\text { 4.1. Web propia } \\
\text { 4.2. Web integrada en la web de la cadena } \\
\text { hotelera }\end{array}$ \\
\hline 5 & Motor de reservas propio & $\begin{array}{l}\text { No tiene motor de reservas propio cuando lo que } \\
\text { incluye es un enlace a booking.com }\end{array}$ \\
\hline 6 & Q de calidad & Indicación en página web \\
\hline 7 & Servicio WIFI & Indicación en página web \\
\hline 8 & Vinculación con cadenas hoteleras & $\begin{array}{l}\text { 8.1. Independientes } \\
\text { 8.2. Vinculado a pequeña cadena } \\
\text { 8.3. Vinculado a gran cadena }\end{array}$ \\
\hline 9 & Uso principales en redes sociales & $\begin{array}{l}\text { 9.1. Facebook propio o de la cadena } \\
\text { 9.2. Twitter propio o de la cadena } \\
\text { 9.3. Linkedln propio o de la cadena } \\
\text { 9.4. Flickr propio o de la cadena. } \\
\text { 9.5. Instagram propio o de la cadena }\end{array}$ \\
\hline
\end{tabular}




\begin{tabular}{|c|c|c|}
\hline & & 9.6. Tuenti propio o de la cadena \\
\hline 10 & Registrado en Google Maps & Verificado o no por el propietario del negocio \\
\hline 11 & TripAdvisor & 12.1 Número de críticas \\
& & 12.2 Puntuación media \\
\hline
\end{tabular}

Fuente: Elaboración propia, adaptado de la literatura científica.

La recolección de datos se ha realizado a través de Microsoft Excel 2010, durante los meses de septiembre y octubre de 2014. Posteriormente se analizaron las variables con el sistema estadístico IBM SPSS 22. LoS gráficos utilizados en los resultados se han realizado a través de Excel.

\section{ANÁLISIS DE LOS RESULTADOS Y DISCUSIÓN}

La discusión de los resultados se ha dividido en tres partes. En primer lugar, el análisis de la implementación de la Web 2.0 en el sector hotelero de Córdoba; seguidamente, se analiza la relación entre el uso de la Web 2.0 en los hoteles y la valoración de los clientes. Por último, se presenta el análisis de la relación entre el uso de la Web 2.0 en los hoteles de Córdoba, la utilización de las redes sociales Facebook y Twitter, las categorías de estrellas y la valoración de los clientes en TripAdvisor.

\subsection{Análisis de la Web 2.0 en los hoteles cordobeses}

La figura 2 muestra la vinculación de cada uno de los hoteles analizados durante esta investigación, observándose que el $46 \%$ de los mismos pertenecen a una gran cadena hotelera, un $31 \%$ son independientes y el $23 \%$ restante pertenecen a una pequeña cadena hotelera. Cabe destacar que estar vinculado a una gran cadena hotelera o, por el contrario, formar parte de una pequeña compañía hotelera o ser independiente puede influir en el uso de las redes sociales, debido a que las pequeñas cadenas y los hoteles independientes toman sus propias decisiones a la hora de elegir las redes sociales a utilizar por el alojamiento, frente a las grandes compañías hoteleras que deben seguir un procedimiento burocrático que, en muchas ocasiones, lleva mucho tiempo para la tramitación de los mismos.

Figura 2. Vinculación de los hoteles

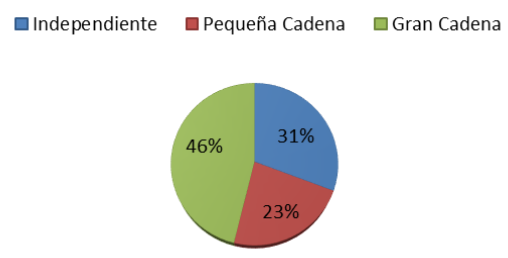

Fuente: Elaboración propia.

Por su parte, las redes sociales o social media están teniendo una gran importancia en Internet para el turismo (Bizirgianni y Dionysopoulou, 2013; Munar y Steen, 2014). Con referencia a los hoteles de Córdoba, el uso de las redes sociales en los hoteles es alto, aunque depende de la red social de la que se hable (figura 3). En este sentido, se puede observar la cantidad de hoteles que se localizan en cada una de la red, destacando Facebook $(92,3 \%)$ como la red social más utilizada por los hoteles de Córdoba, seguida de Twitter (85\%), Linkedln (62\%), Flickr (42\%), Instagram (23,1\%) y Tuenti (23,1\%). En este sentido, solamente el 35\% de los hoteles de Córdoba tienen Facebook propio, por un 57\% que tiene Facebook, pero corresponde a su cadena, y un $8 \%$ que no tiene cuenta en Facebook. Por tanto, Facebook se consolida como la red social más relevante en los hoteles de Córdoba, soportando de esta forma la hipótesis H1 planteada en esta investigación. Para Rodríguez et al., (2012) se trata de la red social virtual más conocida.

Figura 3. Utilización de las redes sociales en los hoteles de Córdoba

= Propio = Cadena $=$ No tiene

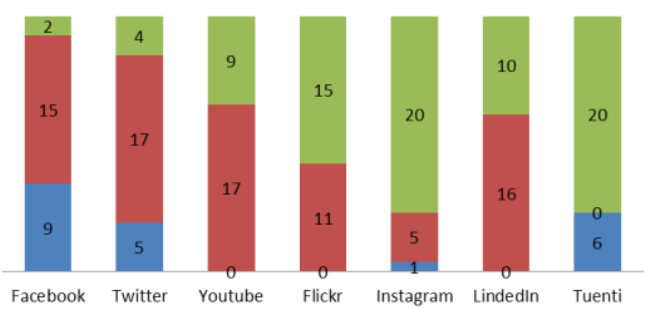

Fuente: Elaboración propia. 
En otro sentido, todos los hoteles analizados cuentan con su página web, contando además con un motor de reservas propio (figura 4). Esta opción supone un coste adicional para los hoteles, pero evita el pago de una comisión a las agencias de viajes intermediarias. En cuanto a la $Q$ de calidad, la mitad de los hoteles la tienen frente a la otra mitad que aún no la disponen (figura 4). En referencia al sistema WIFI, todos los hoteles analizados lo proporcionan gratuitamente, lo cual es apreciado por los clientes, tanto si se encuentran en el hotel por negocios, como si lo hacen por ocio. Por último, todos los hoteles analizados están registrados en Google Maps (figura 4), lo cual también hace que el viaje del cliente sea más fácil, debido que a través de este mapa de Google se puede localizar exactamente donde queda el hotel en la ciudad y cuáles son los recursos turísticos que se encuentran cerca al alojamiento.

\section{Figura 4. Otras variables analizadas}

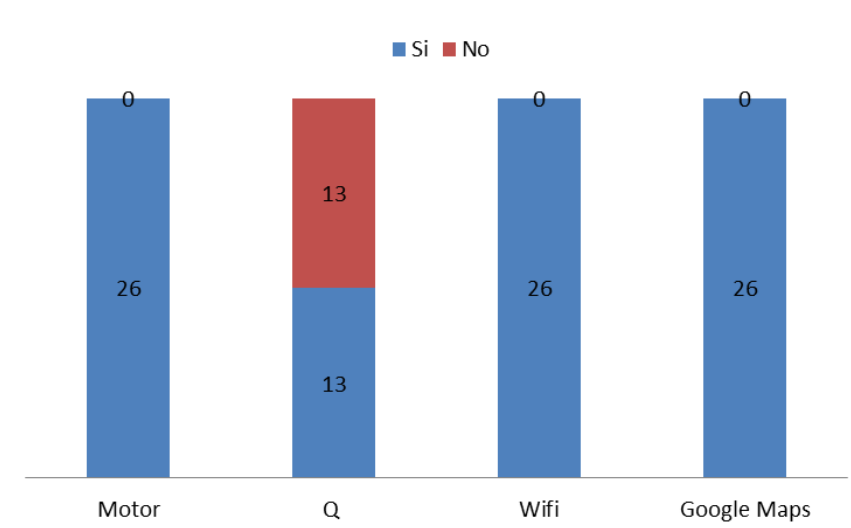

Fuente: Elaboración propia.

Por otro lado, un elemento importante son las fotos y los videos, debido a que los turistas quieren conocer cómo será el posible hotel donde se alojará, y en este sentido, la página web del hotel es la forma más inmediata de acceder a esta información multimedia. En cuanto a los hoteles de Córdoba, más de la mitad de los alojamientos analizados tienen una gran variedad de fotografías en su propia página web (figura 5), frente al resto que tienen disponibles una cantidad más escasa. Referente a los vídeos, el turista también busca información del hotel a través de redes sociales como Youtube u otras que han surgido más recientemente como Vimeo, donde se puede visualizar videos del hotel. En este aspecto, al igual que ocurre con las fotografías, la página web del hotel es el medio por la cual el usuario llega a estos contenidos. Según la figura 6, en la página web de los hoteles de la ciudad de Córdoba son escasos los vídeos, destacando solamente cuatro hoteles con un vídeo cada uno. Por tanto, la mayoría de los hoteles aún no han insertado vídeos, lo que particularmente puede ser negativo para el alojamiento, debido a que al turista le gusta conocer el hotel a través de visitas virtuales.

\section{Figura 5. Numero de fotos de la web del hotel}

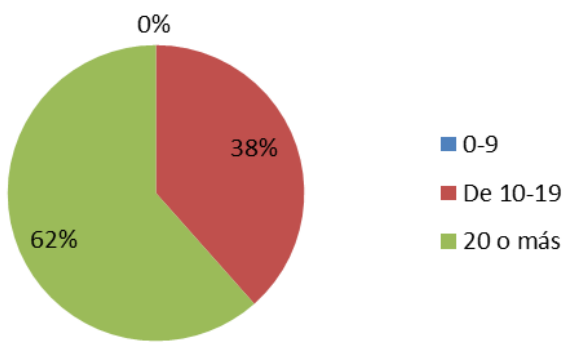

Fuente: Elaboración propia.

Por tanto, en la figura anterior se observa que el $62 \%$ de los hoteles tienen 20 o más fotografías disponibles en su página web para los clientes reales o potenciales. También hay un 38\% que tienen entre 10 y 19 fotos disponibles en su página web. Es decir, el 100\% de los hoteles disponen de fotografías en su página web. 
Figura 6. Número de vídeos en la web de los hoteles

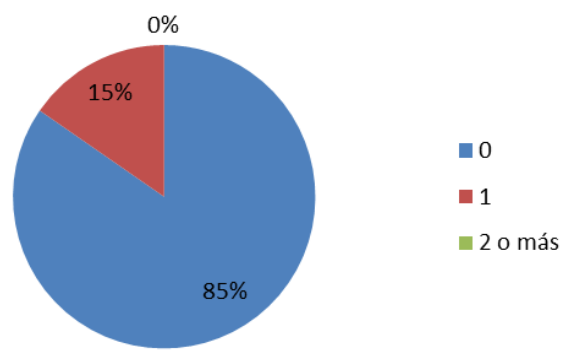

Fuente: Elaboración propia.

En la figura 6 se observa que solo el 15\% de los hoteles de cinco, cuatro y tres estrellas de la ciudad de Córdoba tiene un vídeo disponible para sus clientes reales o potenciales, frente al $85 \%$ que no tienen ningún vídeo.

\subsection{Análisis de la opinión y valoración de los clientes de los hoteles de Córdoba en TripAdvisor}

En el portal web TripAdvisor se ha obtenido una valoración media de 3,99 puntos sobre 5 (tabla 3). Este resultado se ha obtenido al realizar la puntuación media de cada uno de los 26 hoteles analizados. En este sentido, cabe destacar que en TripAdvisor se puntúa en base a 5, frente a otros portales webs que lo hacen en base a 10, como es el caso de Booking, Atrápalo o Rumbo, por citar unos ejemplos. Se debe mencionar que TripAdvisor elimina a su propio criterio todos los comentarios, opiniones y puntuaciones que entienden como irrespetuosas, maleducadas, interesadas, o que no siguen las directrices fijadas por la empresa, lo que puede afectar a la puntuación media de un hotel.

Tabla 3. Valoración media de los hoteles de la ciudad de Córdoba en Tripadvisor

\begin{tabular}{|l|c|c|c|c|}
\cline { 2 - 5 } \multicolumn{1}{c|}{} & Total de Hoteles & Valoración Mínima & Valoración Máxima & $\begin{array}{c}\text { Valoración } \\
\text { Media }\end{array}$ \\
\hline TripAdvisor & 26 & $\begin{array}{c}2,42 \\
(3 \text { estrellas })\end{array}$ & $\begin{array}{c}4,83 \\
(4 \text { estrellas })\end{array}$ & 3,9923 \\
\hline
\end{tabular}

Fuente: Elaboración propia.

Actualmente no existen más de 10 redes sociales realmente relevantes, pero son decenas las páginas webs que recopilan opiniones, valoraciones y puntuaciones de hoteles, cientos los foros de opinión sobre viajes y hoteles y miles los blogs personales, lo que dificultad saber lo que se dice de un hotel en cada momento, para tratar de gestionar su reputación. Por este motivo, puede ser que no sea rentable para el hotel conocer todo lo que gira sobre él en Internet para poder actuar en consecuencia, aunque si debe prestar atención a los primeros resultados que aparecen al escribir el nombre de su hotel en los principales buscadores de Internet, como son Google, Yahoo o Bing (Martínez et al., 2012). En este sentido, para Mashable (2008), existen herramientas gratuitas para las empresas de monitorización online, que tienen la misión de conocer lo que dicen sobre un hotel los expertos, blogueros o simples usuarios.

En el caso en el que se pretenda realizar una gestión optimizada de la reputación online del hotel, se hace necesario acudir a una empresa especializada, bien para encargarle esta tarea o para adquirir un software específico que realice un monitoreo exhaustivo de todo lo que se dice sobre un hotel determinado. Por este motivo existen numerosas empresas especializadas en la gestión de la reputación online de hoteles, como son "Escucha tu cliente", Review Pro, Hotetec, Sidn o Hoteljuice, entre otras. También existen aplicaciones especializadas en monitorizar y gestionar la presencia en redes sociales de las empresas como Social Mention, Google Alerts, Mention, Twazzup, Facebook Insights, Hoot Suite o Addictomatic, entre muchas otras más. Por último, cabe mencionar que los hoteles que tienen mejores comentarios en redes sociales y portales de opiniones, tienen beneficios mayores (Merodio, 2014).

\subsection{Análisis de la relación entre el uso de la Web 2.0 en los hoteles de Córdoba y la valoración de los clientes en TripAdvisor}

Analizados los datos más destacables sobre la Web 2.0 y la reputación de los hoteles de la ciudad de Córdoba en TripAdvisor, se han cogido las variables más destacadas anteriormente, con el objetivo de comprobar si existe correlación entre ellas. En este aspecto, se debe destacar según lo visto anteriormente que no tiene la misma relevancia el tener una página de Facebook o Twitter actualizada, que haber subido algunas fotos a redes sociales como Flickr o Instagram. 
Se han escogido como variables la red social más utilizada por los hoteles de Córdoba: Facebook. También se ha escogido la variable fotos, que destaca sobre los vídeos, y existe un gran número de hoteles con gran cantidad de fotos. Así, un análisis descriptivo de los datos obtenidos indica que existe una correlación directa entre el uso de herramientas de la Web 2.0 y las puntuaciones otorgadas por los consumidores. En concreto, esto surge del análisis de las puntuaciones otorgadas en TripAdvisor, donde la puntuación media obtenida por los 26 hoteles fue de 3,99 sobre 5, aunque como vemos en la tabla 4, la puntuación media varía según el número de estrellas del hotel.

Tabla 4. Puntuación media en Tripadvisor según categoría

\begin{tabular}{|c|c|c|c|}
\hline Estrellas & Media & Número de Hoteles & Desviación estándar \\
\hline 3 & 3,8544 & 9 & 0,68259 \\
\hline 4 & 4,0381 & 16 & 0,46931 \\
\hline 5 & 4,5000 & 1 & \\
\hline TOTAL & 3,9923 & 26 & 0,54749 \\
\hline
\end{tabular}

Fuente: Elaboración propia.

En la tabla anterior se puede observar que la puntuación media de los hoteles de tres estrellas fue de 3,85 puntos, la de cuatro estrellas de 4,03 puntos y la de cinco estrellas de 4,5 puntos. Por tanto, se soporta la hipótesis $\mathrm{H} 4$ de esta investigación, la cual dice que la categoría de los hoteles influye en la puntuación media otorgada por los clientes. Por otro lado, los hoteles con página propia de Facebook, obtienen una valoración media mayor en TripAdvisor que los que tienen Facebook de la cadena o no tienen (tabla 5).

Tabla 5. Puntuación de los hoteles según la propiedad de la página de Facebook

\begin{tabular}{|c|c|c|c|}
\hline Facebook & Media & Número de Hoteles & Desviación estándar \\
\hline Propio & 4,2222 & 9 & 0,46853 \\
\hline Cadena & 4,0207 & 15 & 0,37099 \\
\hline No tiene & 2,7450 & 2 & 0,45962 \\
\hline TOTAL & 3,9923 & 26 & 0,54749 \\
\hline
\end{tabular}

Fuente: Elaboración propia.

Por tanto, se puede observar que los hoteles con página de Facebook propia tienen una valoración de 4,22 frente al 4,02 que tienen aquellos hoteles con página de Facebook perteneciente a la cadena. Por su parte, los hoteles que no tienen Facebook tienen una valoración de 2,74 puntos. Por tanto, las hipótesis H2 (los hoteles que no tiene Facebook son peor valorados que los que sí tienen) y $\mathrm{H} 3$ (los hoteles independientes de cadenas hoteleras tienes valoraciones más altas que los hoteles pertenecientes a una cadena hotelera) son soportadas. Si nos centramos en las críticas recibidas en TripAdvisor, los hoteles con Facebook tienen un mayor número que los que no tienen (tabla 6), destacando las cadenas hoteleras.

Tabla 6. Críticas de los hoteles según la propiedad de la página de Facebook

\begin{tabular}{|c|c|c|c|}
\hline Facebook & Media de críticas & № de Hoteles & Desviación estándar \\
\hline Propio & 252,556 & 9 & 151,3903 \\
\hline Cadena & 420,067 & 15 & 252,4756 \\
\hline No tiene & 51,500 & 2 & 10,6066 \\
\hline TOTAL & 333,731 & 26 & 237,1751 \\
\hline
\end{tabular}

Fuente: Elaboración propia.

Según la tabla anterior, aquellos hoteles con Facebook que pertenecen a la cadena hotelera son los que reciben una media de críticas más elevada $(420,067)$ frente a lo que tienen Facebook propio $(252,556)$. Los que no tienen Facebook reciben muy pocos comentarios en TripAdvisor $(51,500)$. Por tanto, los hoteles con página de Facebook reciben más comentarios en TripAdvisor que aquellos hoteles que no tienen aún esta red social.

Si hacemos el mismo análisis de la tabla 6 entre los hoteles con Twitter obtenemos resultados muy parecidos (tabla 7). De esta manera, los hoteles con Twitter propio tienen una valoración de 4,29 sobre 5 frente al 3,97 de aquellos que tienen dicha red social pero pertenece a la cadena hotelera. Por su parte, los que no tienen Twitter tienen una valoración de 3,69 sobre 5 . 
Tabla 7. Puntuación de los hoteles según la propiedad de la página de Twitter

\begin{tabular}{|c|c|c|c|}
\hline Twitter & Media & № de Hoteles & Desviación estándar \\
\hline Propio & 4,2960 & 5 & 0,30664 \\
\hline Cadena & 3,9724 & 17 & 0,38864 \\
\hline No tienen & 3,6975 & 4 & 1,13802 \\
\hline TOTAL & 3,9923 & 26 & 0,54749 \\
\hline
\end{tabular}

Fuente: Elaboración propia.

Igual ocurre con los hoteles con gran número de fotografías en su página web, que también obtienen valoraciones medias superiores al resto de hoteles con menos fotografías (tabla 8). Así, lo hoteles con un número de fotografías oscilado entre 10 y 19 tienen una valoración de 3,71 puntos sobre 5 frente a los hoteles con 20 o más imágenes, que obtienen una puntuación media de 4,16 sobre 5 .

Tabla 8. Puntuaciones en Tripadvisor según cantidad de fotografías en la web

\begin{tabular}{|c|c|c|c|}
\hline Fotos & Media & № de Hoteles & Desviación estándar \\
\hline $10-19$ & 3,7100 & 10 & 0,43344 \\
\hline 20 o más & 4,1687 & 16 & 0,54820 \\
\hline TOTAL & 3,9923 & 26 & 0,54749 \\
\hline
\end{tabular}

Fuente: Elaboración propia.

Según las tablas anteriores, se puede llegar a la conclusión inmediata de que si un hotel utiliza herramientas de la Web 2.0, es más probable que los huéspedes valoren mejor sus servicios e instalaciones, aunque esto puede ser erróneo, debido a que ningún turista tiene en cuenta ese tipo de factores a la hora de manifestar su grado de satisfacción, pero, si es probable que el uso de la Web 2.0 haya favorecido la elección del hotel, pero nunca hará que valore mejor aspectos como la calidad del servicio, la limpieza e higiene o la cordialidad de los recepcionistas, entre muchos otros elementos. Así, para Martínez et al. (2012), la correlación se debe interpretar de otra forma, identificando qué hoteles muestran mayor preocupación por prestar un servicio de calidad, buscando la mejora continua y adaptándose a las nuevas demandas de los clientes, para mejorar su satisfacción. En el lado opuesto, habría que identificar a aquellos hoteles que apuestan por seguir dando un servicio adecuado a sus clientes, basándose en la experiencia que les ha funcionado durante años, evitando incluir cambios en sus modelos de gestión, comercialización y marketing.

\section{CONCLUSIONES}

Las páginas webs y las redes sociales son herramientas que ayudan a mejorar la promoción de las empresas y, por tanto, la importancia de Internet para las organizaciones es cada vez más relevante. Para las empresas turísticas esto no pasa desapercibido, e incluso, debido a su importancia se ha adoptado el termino Turismo 2.0 para referirse a las herramientas Web 2.0 en el turismo. Por tanto, la presencia de las empresas turísticas (hoteles, agencias de viaje, restaurantes, etc.) en Internet es cada vez mayor, tanto con la página web tradicional como a través de las redes sociales (Facebook, Twitter, Youtube, Lindkedln, etc.).

Entre las conclusiones más relevantes de este artículo sobre los hoteles de la ciudad de Córdoba, se ha podido comprobar que toda la muestra analizada tiene página web, aunque no todos usan las redes sociales. En este aspecto, Facebook es la red social más utilizada en los hoteles de esta ciudad andaluza. Aun así, algunos hoteles ignoran aún la importancia de las redes sociales para la empresa, quedándose solamente con la página web. Este estudio también ha muestra que los hoteles que tienen Facebook propio tienen mayor reputación online que aquellos que tiene un Facebook de la cadena hotelera, o simplemente no tiene. Esto también ha sido demostrado con la red social Twitter. También, se destaca que mientras más fotos se tengan en la página web, la reputación online es mayor. Aunque, cabe destacar que el uso de la Web 2.0 puede haber favorecido la elección del hotel por parte del cliente, pero nunca ha hecho que valore mejor aspectos relacionados con la calidad del servicio, la limpieza e higiene o la cordialidad de los recepcionistas, entre muchos otros elementos. También es importante resaltar de esta correlación entre Facebook y la puntuación en TripAdvisor, que la preocupación del hotel por cuidar su presencia en la Web 2.0 viene de la mano de trasmitir una buena imagen a sus huéspedes, mejorándola constantemente, debido a que la satisfacción del cliente ayudará a que éste valore el hotel en Internet de una forma más positiva, lo cual beneficia a la empresa, aunque a veces esto no se ve reflejado en las valoraciones de los turistas en las Web 2.0 o foros de viajes.

Por tanto, con redes sociales como Facebook o Twitter, los hoteles, además de mejorar su promoción como alojamiento, pueden mejorar la interacción entre los diferentes clientes reales y potenciales. Los resultados de esta investigación pueden ser útiles a los directores de hoteles, puesto que pueden tomar medidas sobre cómo 
mejorar su presencia en Internet, lo cual, unido a un buen servicio al cliente y presentación de los servicios, ayuda a potenciar una buena reputación, tanto offline como online.

Las limitaciones de este estudio hacen referencia a que la amplitud de la población puede resultar poco significativa en algunos aspectos, tanto por el número de hoteles estudiados (se utilizaron solamente lo que aparecen en la página web de turismo de Córdoba). Otra limitación viene por la poca información obtenida de los hoteles en la página web de turismo de Córdoba, con un error como el número total de hoteles de 5 estrellas, debido a que aparece que solo hay uno, cuando realmente existen dos en la ciudad (la página web de turismo de Córdoba lo incorpora en el grupo de hoteles de 4 estrellas). Como líneas futuras de investigación, se encuentra la realización de un estudio similar en otros destinos, con el objetivo de comprobar que las conclusiones obtenidas se cumplen en cualquier destino. Por otra parte, resultaría interesante realizar una nueva toma de datos pasado un tiempo con la misma base de hoteles, y relacionarlo con otras redes sociales.

\section{REFERENCIAS BIBLIOGRAFÍCAS}

Álvarez Rodríguez, M.L.; Mari Pellón, D. y Domínguez Quintas, S. (2010). "Reputación y responsabilidad desde webs corporativas". Área Abierta, 26.

Antón, E. (2008). "Reputación Corporativa Online: Beneficios para las empresas". Prestigia online. Disponible en http://www.prestigiaonline.com/blog/wp-content/uploads/2008/09/reputacion-online.pdf

Aula, P. (2011). "Meshworked reputation: Publicists' views on the reputational impacts of online communication". Public Relations Review, 37 (1): 28-36.

Ayeh, J. K.; Au, N. y Law, R. (2013). "Do We Believe in Trip Advisor? Examining Credibility Perceptions and Online Travelers' Attitude toward Using User-Generated Content”. Journal of Travel Research, 52 (4): 437-452.

Bizirgianni, I. y Dionysopoulou, P. (2013). "The Influence of Tourist Trends of Youth Tourism through Social Media (SM) \& Information and Communication Technologies (ICTs)". Procedia, Social and Behavioral Sciences, 63: 652-660.

Bulchand-Gidumal, J.; Melián-González, S. y González Lopez-Valcarcel, B. (2013). "A social media analysis of the contribution of destinations to client satisfaction with hotel". International Journal of Hospitality Management, 35: 44-47.

Callarisa, L.; Sánchez García, J.; Cardiff, J. y Roshchina, A. (2012). "Harnessing social media platforms to measure customer-based hotel brand equity". Tourism Management Perspectives, 4: 73-79.

Cao, Q. y Schniederjans, M. J. (2006). "Agent-mediated architecture for reputation-based electronic tourism systems: A neural network approach". Information \& Management, 43 (5): 598-606.

Caruana, A. y Ewing, M. T. (2010). "How corporate reputation, quality, and value influence online loyalty". Journal of Business Research, 43 (9-10): 1103-1110.

Castillo Canalejo, A. M.; López-Guzmán, T. y Millán Vázquez De La Torre, M. G. (2010). "El turismo industrial minero como motor de desarrollo en áreas geográficas en declive. Un estudio de caso". Estudios y Perspectivas en Turismo, 19: 382-393.

Celaya, J. (2008). "La empresa en la web 2.0: El impacto de las redes sociales y las nuevas formas de comunicación online en la estrategia empresarial”. Barcelona: Gestión 2000.

Claver Cortés, E.; Pereira Moliner, J. y Molina Azorín, J. F. (2007). "Impacto del tamaño, el tipo de gestión y la categoría sobre el desempeño de los hoteles españoles". Cuadernos de Turismo, 19: 27-45.

Chen, Y.; Mak, B. y Li, Z. (2013). "Quality deterioration in package tours: The interplay of asymmetric information and reputation". Tourism Management, vol. 38: 43-54.

Conde Pérez, E.; Schmidt Cornejo, N.E.C. y Ochoa Llamas, I. (2011). "El turismo electrónico, una necesidad para las empresas del sector". TURyDES, Revista de Investigación en Turismo y Desarrollo Local, 4 (9).

Del Fresno Garcia, M. (2011). "Cómo investigar la reputación online en los medios sociales de la web 2.0". Cuadernos de comunicación Evoca, 5 (1): 29-33.

Del Fresno Garcia, M. (2012). "El consumidor social: Reputación online y social media”. Barcelona: Universitat Oberta de Catalunya.

Dellarocas, C. (2003). "The digitization of word of mouth: promise and challenges of online feedback mechanisms". Management Science, 49 (10): 1407-1424.

Devis-Botella, R. (2010). "Estrategia comercial en redes sociales". Harvard-Deusto: Marketing \& Ventas, 96: 6472.

Domínguez Vila, T. y Araujo Vila, N. (2012). "El fenómeno 2.0 en el sector turístico. El caso de Madrid 2.0". PASOS, Revista de Turismo y Patrimonio Cultural, 10 (3): 225-237. 
Donaire, J. A. y Galí, N. (2011). "La imagen turística de Barcelona en la comunidad Flickr". Cuadernos de Turismo, 27: 291-303.

Escobar-Rodríguez, E. y Carvajal-Trujillo, E. (2013). "An evaluation of Spanish hotel websites: Informational vs. relational strategies". International Journal of Hospitality Management, 33: 228-239.

Foro De Reputación Corporativa (2005). "Introducción a la reputación corporative". Disponible en: http://mouriz.files.wordpress.com/2007/06/introduccion-a-la-reputacion-corporativa.pdf

Fumero, A. y Roca, G. (2007). "Web 2.0. España: Fundación Orange España”. Disponible en: http://fundacionorange.es/areas/25_publicaciones/WEB_DEF_COMPLETO.pdf

González Santa Cruz, F.; Sánchez Cañizares, S. M. y López-Guzmán, T. (2011). "Satisfacción laboral como factor crítico para la calidad: El caso del sector hostelero de la provincia de Córdoba-España”. Estudios y perspectivas en turismo, 20 (5): 1047-1068.

Gretzel, U. y Yoo, K. (2008). "Use and impact of online travel reviews". Information and Communication Technologies in Tourism, 35-46.

Gray, E.R. y Balter, J.M.T. (1998). "Managing image and corporate reputation". Long Range Planning, 31 (5): 685-692.

Gutiérrez, A. M. (2005). "Marketing en Internet. Estrategia y empresa”. Madrid: Pirámide.

Hernández Estárico, E.; Fuentes Medina, M. L. y Morini Marrero, S. (2012). "Una aproximación a la reputación en línea de los establecimientos hoteleros españoles”. Papers de turisme, 52: 63-88.

Hizaji, O. (2002). "E-marketing, una inversión con retorno”. Marketing y Ventas, 50: 6-11.

Hsu, Y. L. (2012). "Facebook as international eMarketing strategy of Taiwan hotels". International Journal of Hospitality Management, 31 (3): 972-980.

Hung, Y. H.; Huang, T. L.; Hsieh, J. C.; Tsuei, H. J.; Cheng, C. C. y Tzeng, G. H. (2012). "Online reputation management for improving marketing by using a hybrid MCDM model". Knowledge-Based Systems, 35: 87-93.

Jeacle, I. y Carter, C. (2011). "In Trip Advisor we trust: Rankings, calculative regimes and abstract systems". Accounting, Organizations and Society, 36 (4): 293-309.

Kahn, R.; Leiner, B.M.; Cerd, V.; Clark, D.; Kleinrock, L.; Lynck, D.; Postel, J.; Roberts, L. y Wolff, S. (1997). "The evolution of the Internet as a Global Information system". The International Information \& Library Review, 29 (2): 129-151.

Kim, M. J.; Chung, N. y Lee, C. K. (2011). "The effect of perceived trust on electronic commerce: Shopping online for tourism products and services in South Korea". Tourism Management, 32 (2): 256-265.

Law, R. (2006). "Internet and Tourism - Part XXI: Trip Advisor”. Journal of Travel \& Tourism Marketing, 20 (1): 75 77.

Lee, H. A.; Law, R. y Murphy, J. (2011). "Helpful reviewers in Trip Advisor, an online travel community". Journal of Travel \& Tourism Marketing, 28 (7): 675-688.

Lincoln, S.R. (2009). "Mastering Web 2.0”. London: Kogan Page.

Liu, L. y Munro, M. (2012). "Systematic analysis of centralized online reputation systems". Decision Support Systems, 52 (2): 438-449.

López, J. M.; López, L. M. y Sanz, B. (2009). "Las dimensiones de la búsqueda en la compra online del turista". Administrando en Entornos Inciertos, 16: 45-56.

Lopez-Gúzman, T.; Millán Vázquez De La Torre, M. G.; Sánchez Cañizares, S. M. y Agudo, E. (2007). "Creación de nuevos productos turísticos: el ecoturismo en la provincia de Córdoba". en Jornadas sobre turismo y Sociedad, Córdoba 2007.

López-Guzmán, T. y Sánchez Cañizares, S. M. (2008). "La creación de productos turísticos utilizando rutas enológicas". PASOS, Revista de Turismo y Patrimonio Cultural, 6 (2): 159-151.

López-Guzmán, T.; Sánchez Cañizares, S. M. y Rodríguez García, J. (2009). "Wine routes in Spain: a case study". Tourism, 74 (4): 421-434.

López-Guzmán, T. y Sánchez Cañizares, S. M. (2012). "Culinary tourism in Córdoba (Spain)”. British Food Journal, 114 (2): 168-179.

Luque, T. y Castañeda, J. A. (2007). “Internet y el valor del negocio”. Mediterráneo económico, 11: $397-415$.

Martínez González, J.A. (2011). "Marketing turístico online”. TURyDES, Revista de Investigación en Turismo y Desarrollo Local, 4 (9). 
Martínez María-Dolores, S.M.; Bernal García, J.J. y Pedro Mellinas, J. (2012). "Los hoteles de la Región de Murcia ante las redes sociales y la reputación online" Revista de Análisis Turístico, 13: 1-10.

Martínez María-Dolores, S.M.; Bernal García, J.J. y Pedro Mellinas, J. (2013). "Análisis del nivel de presencia de los establecimientos hoteleros en la región de Murcia en la web 2.0". Cuadernos de Turismo, 31: 245261.

Mashable (2008). "Top 10 Free Tools for Monitoring Your Brand's Reputation". Disponible en http://mashable.com/2008/12/24/free-brand-monitoring-tools/

Merodio, J. (2014). "Los hoteles con mejor reputación online obtienen mayores beneficios económicos". Disponible en: http://www.juanmerodio.com/2014/hoteles-mejor-reputacion-online-mayores-beneficioseconomicos/

Miguéns, J.; Baggio, R. y Costa, C. (2008). "Social media and tourism destinations: Trip Advisor case study". Advances in Tourism Research, Aveiro (Portugal), Mayo 2008: 1-6.

Millán, G.; Arjona, J. M. y Amador, L. (2014). "A new market segment for olive oil: olive oil tourism in the South of Spain”. Agricultural Sciences, 5 (3): 179-185.

Millán Vázquez De La Torre, M. G. y Agudo Gutiérrez, E. M. (2013). "El Turismo Rural en el Parque Natural de la Sierra Cardeña-Montoro-España: un estudio econométrico". Revista Turismo em Análise, 28 (2): 166183.

Millán Vázquez De La Torre, G. M.; Morales-Fernández, E. J. y Naranjo, L. M. P. (2012). "Análisis del Turismo Gastronómico en la Provincia de Córdoba". Tourism \& Management Studies, 8: 78-87.

Moral Cuadra, S. y Orgaz Agüera, F. (2014). "Las tipologías de turismo en Córdoba (España): una revisión de la literatura científica". Gran Tour, Revista de Investigaciones Turísticas, 9: 112-133.

Moral Cuadra, S.; Cañero Morales, P.; Orgaz Agüera, F. y López-Guzmán, T. (2014). "Una aproximación al oleoturismo en Andalucía, España”. International Journal of World of Tourism, 1 (2): 29-40.

Moreno López, J. A. (2012). “El parque arqueológico de Torreparedones (Baena, Córdoba): un proyecto de desarrollo turístico y cultural”. I Congreso Internacional El patrimonio cultural y natural como motor de desarrollo: investigación e innovación, Universidad Internacional de Andalucía, Jaén 2011: 1048-1062.

Munar, A.M. y Steen Jacobsen, J.K. (2014). "Motivations for sharing tourism experiences through social media". Tourism Management, 43: 46-54.

Murolo, N.L. (2009). "Nuevas pantallas frente al concepto de televisión: Un recorrido por usos y formatos". Razón y palabra, 69 .

O'connor, P. (2008). "User-generated content and travel: A case study on Tripadvisor.com". En O'Connor, P.; Höpken, W. y Gretzel, U.: "Information and communication technologies in Tourism", Proceedings of the International Conference in Innsbruck, Austria, 2008, pp. 47-58. Vienna (Austria): Springer Vienna.

O’Reilly, T. (2005). "What is Web 2.0". Disponible en http://oreilly.com/web2/archive/what-is-web-20.html

Ortiz, J. (2013). "Patios de Córdoba: entre "aves de pétalos"”. Escritura pública, 80: 74-77.

Park, N. Y Lee, K. M. (2007). "Effects of online news forum on corporate reputation". Public Relations Review, 33 (3): 346-348.

Piedrola Ortiz, I. y Artacho Ruiz, C. (2011). "El turismo idiomático: una oportunidad de desarrollo local para Córdoba". Revista de Análisis Turístico, 12: 51-61.

Rivero, F. (2006). “Las ventajas de Internet para el marketing y las ventas”. Marketing y Ventas, 21 (211): 8-13.

Rodríguez Antón, J. M.; Alonso Almeida, M. M.; Rubio Andrada, L. y Esteban Alberdi, C. (2008). "Conocimiento y aprendizaje en las grandes cadenas hoteleras españolas en Iberoamérica: Internet como herramienta de aprendizaje organizativo". Cuadernos de Turismo, 21: 135-157.

Rodríguez Darias, A. J.; Diaz Rodríguez, P. y Santana Talavera, A. (2012). "Estrategias de gestión de imagen de destino en Fuerteventura. De los folletos a la intercomunicación". Cuadernos de Turismo, 30: 219-239.

Sanchez Cañizares, S. M.; López-Guzmán, T. y Millán Vazquez De La Torre, G. (2007). "La satisfacción laboral en los establecimientos hoteleros. Análisis empírico en la provincia de Córdoba". Cuadernos de Turismo, 20: 223-249.

Vaquero, A. (2012). "La reputación online en el marco de la comunicación corporarativa. Una visión sobre la investigación de tendencias y las perspectivas profesionales". Adcomunica: Revista Científica de Estrategias, Tendencias e Innovación en Comunicación, 3: 49-63.

Vásquez, C. (2011). “Complaints online: The case of Trip Advisor”. Journal of Pragmatics, 43 (6): 1707-1717. 
Vermeulen, I. E. y Seegers, D. (2009). "Tried and tested: the impact of online hotel reviews on consumer consideration". Tourism Management, 30 (1): 123-127.

Waddock, S. (2000). "The multiple bottom lines of corporate citizenship: Social investing, reputation and responsibility audits". Business and Society Review, 105: 323-45.

Zeng, B. Y Gerritsen, R. (2014). "What do we know about social media in tourism? A review". Tourism Management Perspectives, 10: 27-36. 